\title{
Homotoxicología aplicada en la medicina veterinaria
}

\author{
Homotoxicology applied in veterinary medicine \\ Giratá Hernández Katherin Adriana ${ }^{1}$ y Fuentes Reyes Edgar Edilberto ${ }^{2}$ \\ ${ }^{1} \mathrm{MVZ}$, Unillanos y ${ }^{2} \mathrm{MVZ}$, Esp., MSc, PhD, Docente Unillanos \\ efuentes@unillanos.edu.co
}

Recibido 14 de Noviembre 2016, Aceptado 21 de Abril 2017

\section{RESUMEN}

La medicina homotoxicológica combina la homeopatía con métodos convencionales de la práctica veterinaria para el beneficio del paciente, apoyado en la medicina interna, utiliza varias técnicas reconocidas como la ecografía, radiología y hematología entre otras, para obtener un diagnóstico adecuado. Bajo esta disciplina se considera que la enfermedad es causada por homotoxinas, las cuales son aquellos factores que alteran la salud del animal, pero que no actúan igual en todas las especies animales y razas. El mecanismo de acción de los medicamentos homotoxicológicos se basa en la reacción de asistencia inmunológica, la cual estimula y modula los mecanismos de defensa y reacciones orgánicas naturales, mediante la reestructuración bioquímica de los tejidos; comparada con la medicina alopática. Es así que la homotoxicología ha cambiado el enfoque del plan terapéutico, buscando estimular el organismo para que de forma natural, mediante procesos inflamatorios, y de excreción, entre otros, eliminen las homotoxinas, sin suprimir los signos clínicos que son la respuesta del organismo para combatirlas. Este artículo recopila el uso de la homotoxicología como terapia alternativa en el tratamiento de afecciones del sistema gastrointestinal en caninos y felinos, como lo son la enfermedad periodontal, megaesófago, gastritis, dilatación/vólvulo gástrico agudo, enteritis, colitis, síndrome del intestino impermeable, megacolon, hepatitis activa crónica, colangiohepatitis, cirrosis hepática, pancreatitis aguda y crónica e intervenciones quirúrgicas como la extracción dental. 
Palabras clave: Homotoxina, homeopatia, intestino, estomago, tratamiento.

\begin{abstract}
Homotoxicological medicine combines homeopathy with conventional methods of veterinary practice for the benefit of the patient, supported by internal medicine, uses several recognized techniques such as ultrasound, radiology and hematology, among others, to obtain an adequate diagnosis. Under this discipline it is considered that the disease is caused by homotoxins, which are those factors that alter the health of the animal, but do not act the same in all animal species and races. The mechanism of action of homotoxicological drugs is based on the immunological assistance reaction, which stimulates and modulates defense mechanisms and natural organic reactions, through the biochemical restructuring of tissues; compared with allopathic medicine. It is so the homotoxicology has changed the approach of the therapeutic plan, seeking to stimulate the body so that naturally, through inflammatory processes, and excretion, among others, eliminate the homotoxins, without suppressing the clinical signs that are the body's response to combat them. This article compiles the use of homotoxicology as an alternative therapy in the treatment of gastrointestinal system disorders in canines and felines, such as periodontal disease, megaesophagus, gastritis, dilatation/acute gastric volvulus, enteritis, colitis, impermeable bowel syndrome, megacolon, chronic active hepatitis, cholangiohepatitis, liver cirrhosis, acute and chronic pancreatitis and surgical procedures such as dental extraction.
\end{abstract}

Keywords: Homotoxina, homeopatia, intestino, estomago, treatment.

\title{
RESUMO
}

A medicina homeopatia homotoxicológico combina métodos convencionais de prática veterinária para o benefício do paciente, suportado na medicina interna, usa várias técnicas conhecidas, tais como ultra-sonografia, radiologia e hematologia entre outros, para obter um diagnóstico correcto. Sob esta disciplina, se considera que a doença é causada por homotoxinas, que são os factores que influem sobre a saúde do animal, mas não actuar o mesmo em todas as espécies 
animais e raças. O mecanismo de ação da drogas homotoxicológicas é baseado na reação imunológica de suporte, que estimula e regula o mecanismos de defesa e reações orgánicas naturais, através de uma reestruturação bioquímica dos tecidos; em comparação com a medicina alopática. É assim que a homotoxicologia tem mudado o foco do plano terapêutico, buscando estimular o organismo para que de forma natural, através de processos inflamatórios, e de excreção, entre outros, eliminam o homotoxinas, sem suprimir sinais clínicos que são a resposta do organismo para combatê-las. Este artigo recolhe o uso da homotoxicologia como uma terapia alternativa para o tratamento de perturbações do tracto gastrointestinal em cães e gatos, tais como a doença periodontal, megaesófago, gastrite, dilatação/vólvulo gástrico agudo, enterite, a colite, síndroma do intestino impermeável, megacólon, hepatite crónica activa, colangiohepatitis, cirrose hepática, pancreatite aguda e crónica e procedimentos cirúrgicos, como extracção dentária.

Palavras-chave: Homotoxina, homeopatia, intestino, estômago, tratamento.

\section{INTRODUCCIÓN}

La homotoxicología es un nuevo concepto terapéutico, de la medicina biológica, creado por el médico alemán Hans Heinrich Reckeweg en el año de 1952, quien realizó estudios sobre la mezcla de sustancias homeopáticas en distintas diluciones, lo cual permite actuar en varios niveles del sistema humoral, celular, molecular y energético (Cooper, 2010), creando así crea una terapia integrativa entre la homeopatía y la medicina convencional (alopática), en la que se contempla la enfermedad como una respuesta del organismo a factores tóxicos, también denominados homotoxinas. Este concepto de "enfermedad" ha cambiado el enfoque del plan terapéutico, porque se fundamenta en la estimulación del organismo, para que de forma natural, como son los procesos inflamatorios y de excreción, entre otros, se eliminen estas toxinas, lo que difiere del enfoque que tiene la medicina alopática, la cual busca suprimir los síntomas, que no son sino la expresión natural del organismo para combatirlas (IHA, 2007d). Teniendo en cuenta lo anterior en la medicina convencional, por ejemplo el uso indiscriminado 
de medicamentos como los antibióticos, ha generado en varios casos aumento de la resistencia de patógenos a la acción de estos fármacos (Zepeda, 1998), adicionalmente, de acuerdo al tratamiento, los tiempos de retiro debido a su residualidad, provocando efectos secundarios, contraindicaciones e interacciones con otros medicamentos y sus posibles efectos negativos sobre el medio ambiente (Murillo, 2010).

Con el tiempo el empleo de terapias alternativas o integrativas se ha venido difundiendo ampliamente en la medicina humana y afortunadamente en la medicina veterinaria, puesto que se recurre a la homeopatía cuando las terapias convencionales no pueden solucionar el problema, o en el caso de tratamientos prolongados que terminarían lesionando algún órgano por efectos secundarios de algunos medicamentos, situación que no se presenta con las sustancias homotoxicológicas, las cuales utilizan diferentes diluciones que actúan a nivel celular sin generar efectos nocivos (IHA, 2007h); por lo tanto la medicina homotoxicológica se puede aplicar en medicina veterinaria, puesto que alrededor del $24 \%$ al $28 \%$ de las consultas a las clínicas veterinarias son para el tratamiento de afecciones del sistema gastrointestinal (Almanza et al., 2007); además este sistema es de gran importancia porque cumple funciones de absorción y al tiempo excreta homotoxinas que puedan afectar la integridad de su revestimiento epitelial y generar no solamente enfermedad local sino sistémica (IHA, 2007b).

Es así, que el objetivo de esta recopilación es contextualizar sobre el uso de la homotoxicología en el tratamiento de diferentes afecciones en pequeños animales, describiendo las indicaciones y el mecanismo de acción del uso de sus medicamentos que son empleados en la medicina veterinaria.

\section{HOMOTOXICOLOGÍA}

El termino homotoxicología procede de tres raíces del latín homo (hombre), y de los griegos toxikon (veneno) y lógos (tratado), por lo cual significa el estudio de la influencia de las sustancias tóxicas sobre los seres humanos (IHA, 2007c), ciencia que aunque inicialmente fue desarrollada para la medicina humana varios de sus 
conceptos y terapéuticas son seguras y eficaces para ser aplicados en veterinaria (García et al., 2003b).

La homotoxicología estudia las enfermedades y el tratamiento biológico de las mismas, partiendo de los principios fundamentales de la homeopatía (Torres, 2009); por lo tanto, también es conocida como homeopatía de segunda generación, apoyada en un concepto intermedio entre la homeopatía y la medicina convencional (Tabla 1), aplicando un sólido diagnóstico de la alopatía y un tratamiento suave y no tóxico de la homeopatía, además se fundamenta en la medicina interna, utilizando técnicas de diagnóstico como ecografía, radiología, hematología, entre otras (Broadfoot et al., 2009).

Tabla 1. Relación entre las prácticas convencionales y alternativas en la homotoxicología y homeopatía

\begin{tabular}{cccc}
\hline & Alopatía & Homotoxicología & Homeopatía clásica \\
\hline Diagnóstico & Hallazgos clínicos & Hallazgos clínicos & $\begin{array}{c}\text { Resultados mentales } \\
\text { y somáticas } \\
\text { constitucional }\end{array}$ \\
Terapia & Para tratar los & $\begin{array}{c}\text { Para crear el } \\
\text { síntomas }\end{array}$ & $\begin{array}{c}\text { Para crear el } \\
\text { bienestar general }\end{array}$ \\
bedicamentar general \\
\end{tabular}

Fuente: Broadfoot et al., (2009)

Fue desarrollada por el médico toxicólogo y homeópata Hans Heinrich Reckeweg (1952) quien consideró el principio del fisiólogo Von Bertalanffy, apreciando a todo organismo vivo como un sistema dinámico de flujo, el cual se ajusta continuamente al medio ambiente que lo rodea con el propósito de mantenerse en un estado de equilibrio, generando el concepto de homotoxina, para nombrar las sustancias que son nocivas para el organismo, consideradas la causa de enfermedad, porque son las responsables de alterar la armonía en el funcionamiento del organismo (IHA, 2007c). 
En la homotoxicología es de vital importancia varios aspectos histológicos como el concepto de matriz viviente (MV), antes llamado "medio interno", la cual tiene tres niveles que interactúan entre sí: la matriz extracelular (MEC), la matriz intracelular (MIC) y la matriz intranuclear (MIN); la MV se refiere al entorno directo de la célula tanto estructural como fisiológico (IHA, 2007c). La calidad de vida de la célula se relaciona directamente con la naturaleza de su entorno directo, porque en esta zona toma su alimento y energía y deposita sus productos de desecho (Broadfoot, 2006); por ejemplo en el caso de una infección bacteriana, el agente no es la causa, sino que el medio interno del paciente se convierte en un medio de cultivo que favorece la proliferación del microorganismo (IHA, 2007e).

El objetivo de la homotoxicología es estimular y modular los mecanismos de defensa y reacciones orgánicas naturales, mediante la reestructuración bioquímica de los tejidos celulares, asimismo accionar los sistemas enzimáticos e inmunológicos contra las homotoxinas induciendo su eliminación por las vías humorales (Cian, 2005).

\section{PRICIPIOS DE LA HOMEOPATÍA Y DE LA HOMOTOXICOLOGÍA}

Los principios de estas dos formas de medicina es el resultado de varios estudios realizados por diferentes investigadores, siendo los de la homeopatía: Similitud, pincipio de Burgi, diluciones homeopáticas y ley de Hering (Cuadro 1), y los de la homotoxicología: Homotoxinas, homotoxonas, retoxinas, homotoxicosis y regulación basal (Cuadro 2).

Fases humorales: Aún no están alterados los sistemas intracelulares. El sistema de defensa del animal se halla intacto y puede eliminar las homotoxinas a través de diferentes vías (Fisher y Dantas, 2001), caracterizadas por que el organismo intenta conseguir la detoxificación (eliminación) espontánea (IHA, 2007c) (Cuadro 3). 
Cuadro 1. Principios de la homeopatía incluidos en la homotoxicología

\begin{tabular}{|c|c|}
\hline $\begin{array}{l}\text { Principio de } \\
\text { similitud }\end{array}$ & $\begin{array}{l}\text { Este principio lo estableció Hahnemann en 1796, padre de la } \\
\text { homeopatía, quien después de varios experimentos y observaciones } \\
\text { determinó que un paciente con cuadro clínico parecido al cuadro tóxico } \\
\text { descrito al ingerir concentraciones elevadas de una toxina en estado de } \\
\text { salud, podrá tratarse con dicha toxina en concentraciones muy bajas } \\
\text { para curar su enfermedad, lo cual llamó principio de similitud (De Medio, } \\
2004 \text { ). }\end{array}$ \\
\hline $\begin{array}{c}\text { Principio de } \\
\text { Burgi }\end{array}$ & $\begin{array}{l}\text { Burgi en } 1932 \text { propuso que la administración simultánea de diferentes } \\
\text { sustancias con acciones terapéuticas similares produciría un efecto } \\
\text { sinérgico cuyo efecto sería más que la suma de los efectos individuales } \\
\text { de cada sustancia, fundamentos que se utilizaron para generar los } \\
\text { medicamentos compuestos antihomotóxicos (IHA, 2007c). }\end{array}$ \\
\hline $\begin{array}{l}\text { Diluciones } \\
\text { homeopáticas }\end{array}$ & $\begin{array}{l}\text { En los medicamentos homotoxicológicos solo se emplean diluciones } \\
\text { homeopáticas decimales. La farmacopea alemana (HAB) describe cómo } \\
\text { se realiza este tipo de diluciones: la primera dilución decimal } 1 / 10 \text { (D1) } \\
\text { se elabora a partir de la tintura madre concentrada, y se diluye con } \\
\text { alcohol o agua (diluyente) hasta una medida estándar } 100 \% \text {, luego se } \\
\text { agita con firmeza } 10 \text { veces (dinamizar) y de está } \mathrm{D} 1 \text {, se toma un parte y } \\
\text { se mezcla con nueve partes de diluyente en un frasco nuevo. Este } \\
\text { líquido se dinamiza de nuevo y se crea una dilución D2, y así } \\
\text { sucesivamente (IHA, 2007c). }\end{array}$ \\
\hline Ley de Hering & $\begin{array}{l}\text { Este principio se fundamenta en que las enfermedades evolucionan con } \\
\text { el paso del tiempo: desde órganos y tejidos que no afectan el } \\
\text { funcionamiento basal del organismo hasta otras partes orgánicas que } \\
\text { son importantes para mantener la armonía metabólica, además } \\
\text { molestias que no afectan las células se pueden convertir en } \\
\text { enfermedades citogenéticas. }\end{array}$ \\
\hline
\end{tabular}

Fases matriciales: Las homotoxinas se depositan primero en el retículo de la matriz extracelular (MEC), en una evolución posterior, se alteran tanto sus componentes estructurales como sus funciones (García et al., 2003a). Las fases matriciales son cruciales en la evolución del paciente, es el punto donde se produce el paso real desde la presencia o el efecto extracelular de la homotoxina a la intracelular (IHA, 2007c) (Cuadro 3).

Fases celulares: En estas fases de manera lenta pero segura se inhiben las funciones de la célula hasta el punto de su destrucción, los mecanismos de 
autorregulación fracasan y el organismo intenta compensarlo (Barros, 2011; IHA, 2007d) (Cuadro 3).

Cuadro 2. Principios fundamentales de la homotoxicología

Homotoxinas

Homotoxonas

Retoxinas

Homotoxicosis

Regulación basal
Son todos aquellos factores ambientales, nutricionales, bacterianos, bioquímicos, víricos, entre otros, que causan trastornos en la salud de los animales (García et al., 2003a); es importante señalar que las homotoxinas no son necesariamente sólo los venenos químicos, de hecho, hay muchas cosas que pueden afectar negativamente a los sistemas vivos, cualquier cosa en exceso puede convertirse en un homotoxina (Broadfoot et al., 2009). Las homotoxina se clasifican en endógenas, formadas en el organismo como los productos del metabolismo, y en exógenas, que proceden del medio ambiente, como microbios e injurias entre otros (IHA, 2007i). Los radicales libres son un ejemplo de homotoxina endógena, estos son componentes esenciales del metabolismo celular, pero si se acumula a niveles superiores a los mecanismos de compensación del cuerpo, puede generar daño celular mediante la acción oxidativa directa y alterar otras moléculas y producir nuevos componentes reactivos (Broadfoot et al., 2009).

La formación de homotoxonas es una forma de detoxificación que ocurre a nivel del hígado y del tejido conectivo, considerándose un proceso inmunodefensivo durante las reacciones inflamatorias; esta formación consiste en la unión de dos homotoxinas (HTI), o de una homotoxina con un anticuerpo u otro factor no tóxico, con la finalidad de neutralizar las propiedades tóxicas de las HTI (García et al., 2003a).

También llamados "tóxicos residuales", son los depósitos de homotoxinas con sustancias endógenas, que no pueden eliminarse mediante la excreción o la inflamación (Herrera y Villegas, 2005).

Es el concepto de enfermedad en homotoxicología, es decir, la reacción que genera la presencia de las homotoxinas sobre las funciones de la célula, y a través de estas, sobre la función de todo el organismo. Los signos clínicos se contemplan como la expresión o el intento de los sistemas fisiológicos de defender el organismo, como la excreción (vómito, diarrea), la inflamación (fiebre), la deposición (verrugas, adiposidad), la impregnación (asma), la degeneración (tuberculosis, cirrosis), y la neoplasia, para eliminar las toxinas (Ares y García, 2009; IHA, 2007c).

Es la posibilidad de regulación local del sistema básico unido a los sistemas superiores de regulación nerviosa, celular y humoral; está formado por la sustancia básica (matriz extracelular) y los componentes celulares, humorales y nerviosos (Broadfoot, 2006). 
Cuadro 3. Procesos de las fases de la homotoxicosis

Excreción: El organismo reacciona ante las toxinas a través del aumento de los mecanismos fisiológicos de excreción como sudor, lágrimas, orina, secreciones gastrointestinales, entre otros, para lo cual se activa la detoxificación en hígado, pulmón, riñón, mucosas y piel (Cian, 2005), este estado de hiperexcreción no se considera como una enfermedad en sí (García et al., 2003a), si el cuerpo no puede excretar la homotoxina, o si esta penetra la barrera epitelial, los mecanismos biorreguladores pueden desencadenar la cascada inflamatoria para eliminar la homotoxina

Fases humorales

Fases matriciales

\section{Fases celulares}

Inflamación o reacción: Al no poder eliminar las toxinas extracelulares, empieza la cascada inflamatoria (García et al., 2003a; IHA, 2007c), a través de anticuerpos, respuesta celular, fagocitosis, proteasas y el aumento de la irrigación del área afectada. El exudado inflamatorio pasa a las superficies epiteliales y se elimina al exterior o es absorbido por el torrente sanguíneo y procesados por el sistema linforreticular (Broadfoot et al., 2009). En esta fase los animales suelen presentar edema, alergias y eritema (Cian, 2005).

Depósito: Los mecanismos de excreción están sobrecargados y las toxinas se depositan en la MEC, las células del sistema inmunológico rodean las toxinas para que no se extiendan (García et al., 2003a); los animales suelen presentar pólipos, quistes, verrugas, papilomas, miomas, ateromas, entre otros (Cian, 2005; IHA, 2007a); también es en este periodo los trastornos no son obvios porque el cuerpo lo compensa para que la reacción biológica no sea evidente en el paciente o para el médico, lo cual dificulta un diagnóstico, por los cambios (Broadfoot et al., 2009).

Impregnación: Las homotoxinas están en el interior o exterior de la célula, a nivel del tejido conectivo y la MEC, pero tienen efectos intracelular como: alteración de comunicación, cambios en interfase del impulso nervioso, aporte de oxígeno y sustratos, entre otros (Cian, 2005). Las enfermedades aparecen en crisis, con grandes períodos de latencia, o forma aguda potencialmente mortales como: asma, úlcera gástrica, hepatotoxicidad, hidronefrosis y alergias; con sintomatología de dolor, malestar y fatiga (Broadfoot et al., 2009; IHA, 2007d).

Degeneración: Hay muerte celular, pérdida de tejido y trastornos degenerativos; el sistema inmunológico actúa contra el propio organismo destruyéndose en el intento de auto-organización. Los animales presentan enfermedades degenerativas crónicas, la mayoría irreversibles como: las autoinmunes, cirrosis, artrosis, fibrosis, insuficiencias hepática y renal, entre otras (Cian, 2005; IHA, 2007d).

Neoplasia: Las homotoxinas han penetrado en el núcleo, alterando y dañando el control celular y mecanismos de reparación, hay bloqueo de enzimas, disociación de ADN y alteración de genes (Broadfoot et al., 2009); las células pierden su especificidad y pueden hacer metástasis, produciendo aumento en la tumorogenesis (IHA, 2007d). 


\section{CORTE BIOLÓGICO: DIVISIÓN DE REGULACIÓN / DESREGULACIÓN}

Es una línea imaginaria que separa las tres primeras fases de la enfermedad, entre la fase de depósito y la de impregnación (García et al., 2003a), marca la diferencia entre la acumulación de homotoxinas extracelular y la intracelular o su efecto, entre la autorregulación y la compensación (IHA, 2007d); además separa entre sí los principios terapéuticos y patogenéticos en la homotoxicología, e indica con frecuencia el punto sin retorno (Broadfoot et al., 2009).

Al lado izquierdo del corte biológico se producen todas las enfermedades en las que las homotoxinas son causales o sus efectos son extracelulares, incluyendo patologías de curso agudo; al lado derecho la presencia o el efecto de la homotoxina es principalmente intracelular, produciéndose con frecuencia una lesión irreparable de la célula, se encuentran patologías principalmente crónicas, donde la mayoría de veces la salud del animal se encuentra comprometida (Broadfoot et al., 2009; IHA, 2007d).

\section{Evolución hacia la enfermedad (vicariación progresiva)}

Señala que la evolución del animal tiene un pronóstico de empeoramiento de los síntomas y que las homotoxinas se están acumulando (fase de depósito), es decir de van de la MEC a la matriz intracelular (MIC) en lugar de ser procesadas y eliminadas (IHA, 2007d); se observa una progresión de izquierda a derecha en la línea de corte biológico, lo que significa que las homotoxinas están haciendo más daño al organismo y sus defensas no pueden revertir estos efectos (Broadfoot et al., 2009).

\section{Evolución hacia la salud (vicariación regresiva)}

La salud del animal presenta una mejoría global de los síntomas; las homotoxinas están evolucionando desde los tejidos más profundos hasta la superficie (IHA, 2007d). 


\section{FACTORES QUE INTERVIENEN EN LA ACCIÓN DE LA MEDICINA ANTIHOMOTÓXICA}

El sistema de gran defensa hace referencia a cinco subsistemas que actúan en forma conjunta en lucha contra la acción de las homotoxinas tratando de recuperar el equilibrio que implica la salud (García et al., 2003b) en este sistema se observan procesos de regulación local así como las respuestas coordinadas dentro de la inmunidad celular y humoral con regulación endocrina y neurológica (Cuadro 4 y Figura 1).

Cuadro 4. Mecanismos de acción de los medicamentos homotoxicos y los sistemas implicados en el proceso

Sistema reticuloendotelial: Comprende el sistema mononuclear fagocítico el cual está encargado de realizar funciones como la formación de anticuerpos, fagocitosis y defensa humoral (Fernández y Brugieres, 1997); la reacción antígeno (Ag) anticuerpo (Ac) ocurre a nivel del tejido conectivo, donde el $\mathrm{Ag}$ correspondiente a la homotoxina que se combina con un Ac formando un compuesto no tóxico, la homotoxona (Herrera y Villegas, 2005).

Mecanismo adenohipofisis/adrenal: La defensa humoral procura la eliminación de las homotoxinas mediante la inflamación, la cual está bajo control neuroendocrino (Cian, 2005). La adenohipofisis es estimulada para la liberación de hormonas que activan la corteza suprarrenal que restringirá o inhibirá la respuesta inflamatoria (Brandan et al., 2010).

Defensa neural tóxica: Las homotoxinas a nivel de la MEC pueden estimular las terminales nerviosas activando las fases simpaticotónica, vagotónica y/o parasimpaticotónica, permitiendo respuestas rápidas mediante los síndromes de irritación o excitación debidos a la liberación de neurotransmisores como la acetilcolina y/o adrenalina (Condori et al., 2007).

Detoxificación hepática: El hígado es el principal órgano de detoxificación del organismo, se encarga de modificar químicamente las homotoxinas a través de fenómenos de oxidación, reducción, hidrólisis y conjugación mediante enzimas hepáticas detoxificantes formando compuestos no tóxicos llamados homotoxonas (Angosto, 2008; IHA, 2007a).

Detoxificación en el tejido conectivo: El tejido conectivo abarca un amplio sistema de drenaje, está bajo el control del sistema nervioso autónomo y hormonal mediante el metabolismo acido-base siguiendo un biorritmo específico; recoge productos de desecho expulsados por las células y los envía a través del sistema linfático o por los capilares (en el caso de proteínas), o simplemente las almacena temporalmente en depósitos (García et al., 2003a).

Las células están rodeadas por una sustancia fundamental llamada MEC, que actúa como una red para la recopilación de la información, evaluación y 
transmisión, el fibroblasto es la célula de control funcional de la MEC siendo el centro de la actividad metabólica que controla las actividades que ocurren allí. El sistema neuroendocrino funciona a través de sustancia fundamental para coordinar las funciones corporales (Broadfoot et al., 2009); el sistema de la gran defensa mantiene un equilibrio del $\mathrm{pH}$ a través de la fluctuación de las condiciones de acidez y alcalinidad.

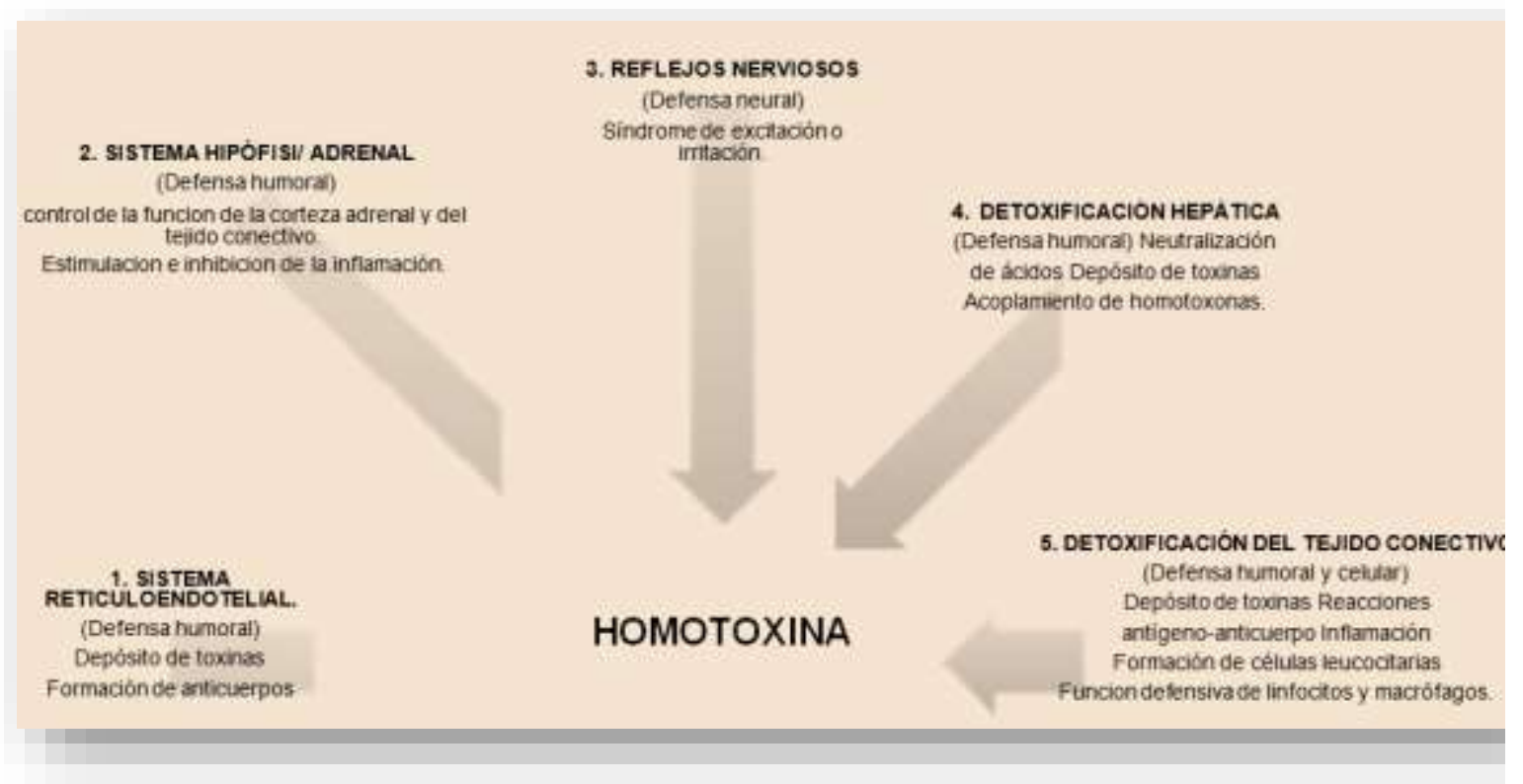

Figura 1. Sistema da la Gran defensa.

Fuente: García et al., (2003a).

Según Heine y Schmolz, (1999) los cambios extremos de $\mathrm{pH}$ permiten la reconstrucción y el mantenimiento de las fibras de colágeno estructural; durante el período ácido las moléculas de colágeno se vuelve más suave y más delgada y la matriz se vuelve más líquida; en cambio durante la fase alcalina los geles de colágeno de la matriz se hinchan, en tales circunstancias las fibras del dolor y otras sustancias inflamatorias se activan, y puede resultar en hiperactividad del sistema inmunológico y daños en los tejidos. En estas situaciones el uso de fórmulas antihomotóxicas es beneficioso para activar los fibrocitos y otros sistemas de autorregulación para ayudar al organismo en la recuperación de la exposición de la homotoxina (Bastidas, 2012). 


\section{Reacción de asistencia inmunológica}

La reacción de asistencia inmunológica representa una teoría de la terapia antihomotóxica, basándose en reacciones por dosis pequeñas de antígenos (potencias bajas: diluciones D1 a D14) (García et al., 2003b); en la Figura 2 se observa la interacción de las diferentes fórmulas homotoxicológicas con el sistema de la gran defensa.

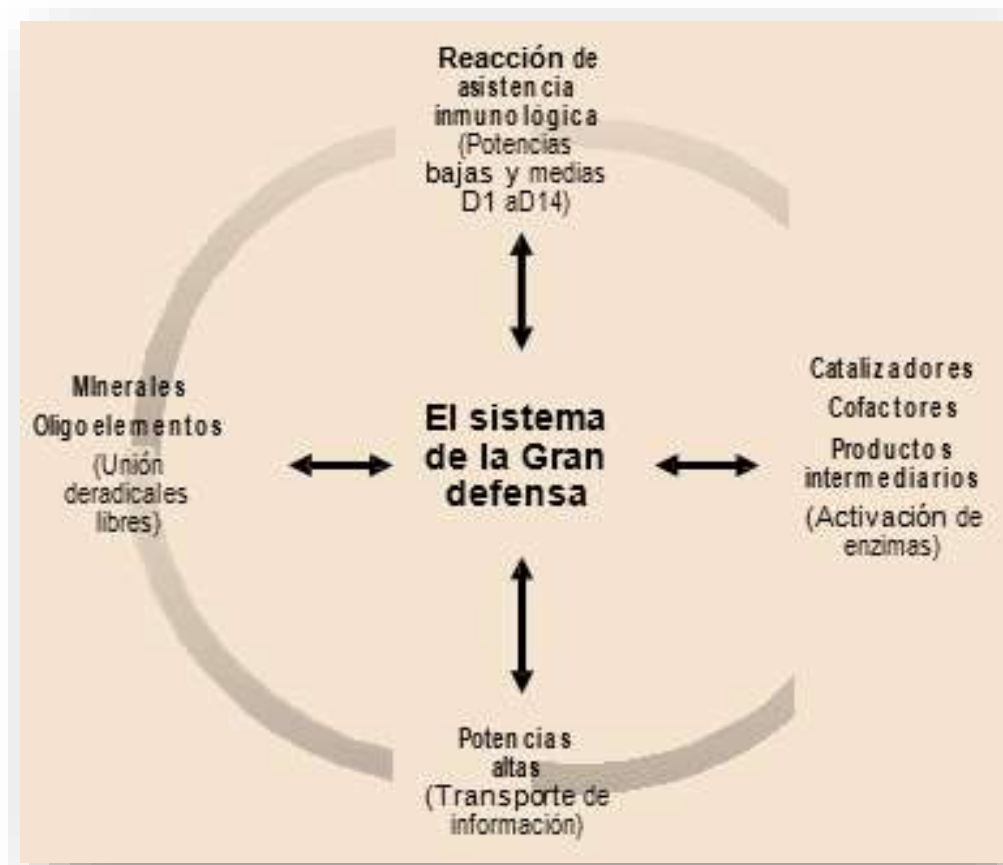

Figura 2. Círculo funcional de la terapia antihomotóxica.

Fuente: Adaptado de García et al., (2003b).

Cuando se administran los preparados antihomotóxicos por cualquier vía (oral, intravenosa, subcutánea), primero se enfrentan a los macrófagos/monocitos, los cuales los degradan a pequeños fragmentos que son mostrados a la superficie celular para el procesamiento del antígeno en forma de epítope, los cuales se unen al complejo mayor de histocompatibilidad $(\mathrm{CMH})$ para ser reconocidos por los linfocitos inmaduros o indiferenciados (LTh0); los LTh0 al tomar estos epítopes se convierten en linfocitos reguladores (LTh3), luego viajan por los ganglios a los nódulos linfáticos más cercanos y allí forman clones celulares con epítope, que se distribuyen por todo el organismo por la circulación sanguínea; en las áreas 
disreguladoras, especialmente las inflamadas, se atraen a los LTh3 por medio de sustancias quimiotácticas como quimiocinas, factores del complemento y otros (Heine y Schmolz, 1999) (Figura 3).

En base a sus epítopes los LTh3 pueden reconocer a los linfocitos proinflamatorios, los T4 (LT4) y sus subpoblaciones: linfocitos ayudadores Th1 (LTh1) y Th2 (LTh2), esto, debido al principio de similitud estimulando a LTh3 para secretar un factor de crecimiento transformante- $\beta$ (TGB- $\beta$ ) y en menor medida interleucina 4 (IL-4) y 10 (IL-10) (Figura 3). El TGB- $\beta$ es la citocina antiinflamatoria más potente del organismo, debido a que impide que los linfocitos proinflamatorios favorezcan la reacción inflamatoria, sumado a esto los LTh2 refuerzan su propia desactivación liberando IL-4 e IL-10, y simultáneamente los linfocitos B (LB) son estimulados para realizar la síntesis de inmunoglobulinas (Hernández y Alvarado, 2001).

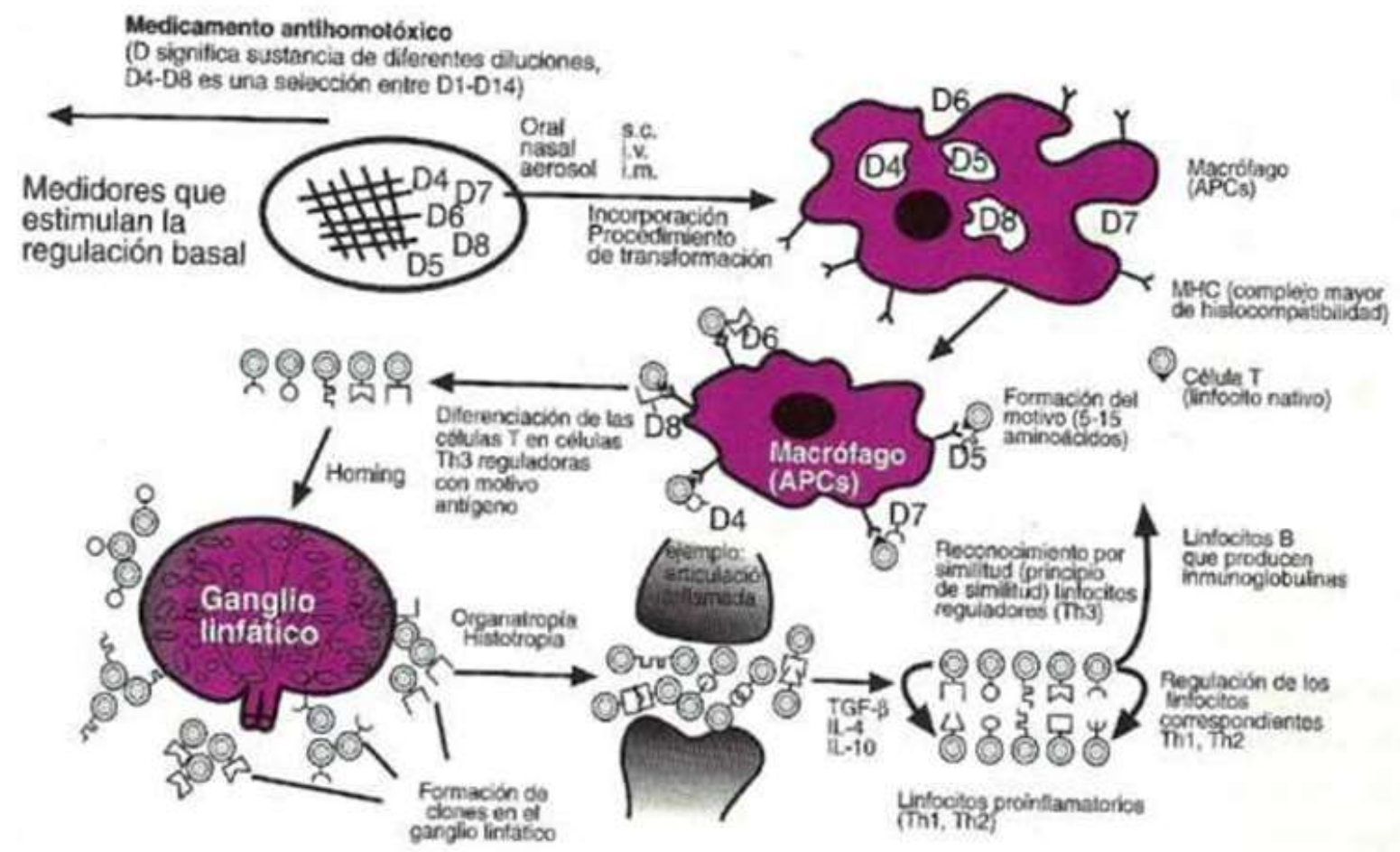

Figura 3. Reacción de asistencia inmunológica y su relación con las fórmulas antihomotóxicas de dosis bajas.

Fuente: Hernández y Alvarado, (2001). 
Los macrófagos son estimulados por diluciones homeopáticas de baja potencia en concentraciones en el rango D1 a D14 y se pueden administrar por diferentes vías, al ser absorbidos estos agentes antihomotóxicos se unen con los macrófagos formando epítopes de antígenos, que son requisitos previos para la respuesta de los linfocitos (Th3), los cuales se encuentran quimiotácticamente con T4, Th1, Th2 por antígenos similares y suprimen las homotoxinas por la liberación de citoquinas (Broadfoot et al., 2009; Heine y Schmolz, 1999).

\section{FUNDAMENTOS DE LA HOMOTOXICOLOGÍA}

Drenaje y detoxificación: El tratamiento en estado de intoxicación va encaminado a eliminar las homotoxinas y no a suprimir la acción del organismo contra estas (ejemplo: la inflamación, considerada la depuración natural de la $\mathrm{MEC}$ ), lo que correspondería al tratamiento sintomático usado en la medicina convencional. Los medicamentos de drenaje y detoxificación actúan sobre el almacenamiento de las toxinas a nivel de la MEC y no a nivel de la MIC, por lo tanto se realiza drenaje y detoxificación al tiempo para dar soporte a los órganos de eliminación (hígado y riñón) y no producir una sobrecarga toxica; al realizar esto se debe suministrar suficientes líquidos para facilitar la eliminación de las toxinas. En casos crónicos, es mejor iniciar con una terapia de apoyo hacia los órganos de eliminación para prepararlos para la llegada de las homotoxinas (IHA, 2007a).

En casos leves, enfermedades ubicadas en las fases de excreción o inflamación, se utiliza el Detox Kit (kit de desintoxicación), para drenar y detoxificar, que consiste en Berberis Homaccord (drenaje del riñón y da soporte hepático y a la función adrenal) y Nux vómica Homaccord (soporte hepático y drenaje del sistema gastrointestinal) como una excelente opción; para casos medianamente leves, aquellas homotoxicosis ubicadas en fases inflamatorias crónicas o de deposición, la terapia consiste en una mezcla de Solidago compositum (soporte y drenaje renal), Hepar compositum (soporte y drenaje hepático), Galium Heel (detoxificación y drenaje celular y reparación orgánica) y Lymphomyosot (drenaje linfático y apoyo inmune). En casos más graves, fases de impregnación y 
desdiferenciación, la fórmula de detoxificación se adapta a las necesidades específicas del paciente. En general esta fórmula incluye Galium Heel, Lymphomyosot, Thyroidea compositum, Solidago compositum, Hepar compositum y/o Hepeel, y catalizadores como Ubichinon compositum, Coenzima compositum y Glioxal compositum, los cuales son dosificados en función de la respuesta del paciente, pudiéndose administrar con poca frecuencia como dos veces por semana y alta frecuencia (varias veces al día); generalmente se prescribe dos 0 tres veces al día (Broadfoot et al., 2009).

Inmunomodulación: El sistema inmunitario se utiliza en el tratamiento de enfermedades crónicas y se busca mantener los signos clínicos de inflamación en niveles aceptables para el paciente, o estimular un sistema inmunitario no reactivo; en esta inmunomodulación intervienen varias células como los linfocitos TH0, TH1, TH-2 y TH-3; células dendríticas, células presentadoras de antígeno; esta herramienta terapéutica busca mantener un equilibrio en los linfocitos $\mathrm{TH}-1$ y TH-2 (IHA, 2007a).

Apoyo orgánico y celular: Se utiliza en caso de enfermedades crónicas y/o degenerativas, junto con los dos primeros se busca producir una mejoría fisiológica del tejido con el apoyo de las células aumentando su oxigenación, lo cual optimiza las funciones orgánicas, tratando de mejorar el órgano como "unidad". El suministro energético de una célula depende del ciclo de Krebs o ciclo del ácido cítrico, donde intervienen catalizadores que hacen el papel de potencializar, se pueden utilizar dos estrategias: el paquete combinado (catalizadores) o dos medicamentos compositum específicos (Coenzyme compositum y Ubichinon compositum); para dar soporte orgánico se han desarrollado medicamentos compositum especiales, existiendo en el mercado una fórmula para casi todos los órganos como Mucosa compositum (Mucosa); Hepar compositum (hígado); Tonsilla compositum (Sistema linfático); Pulsatilla compositum / Echinacea compositum (Sistema de defensa); Placenta compositum (circulación) (IHA, 2007h). 


\section{TERAPIA ANTIHOMOTÓXICA}

La terapia antihomotóxica busca establecer la homeostasis biológica mediante la estimulación de los mecanismos de regulación propios del organismo; se parte del corte biológico para diferenciar las enfermedades que están al lado derecho o izquierdo, y así emplear los tres fundamentos de la homotoxicología: Drenaje y detoxicación. inmunomodulación y apoyo orgánico y celular (Smit, 2004).

Las fases de la izquierda de la división biológica se asocian a la recuperación completa si se estimula correctamente el mecanismo defensivo del animal y se consigue un drenaje y una detoxificación adecuados; a la derecha el tratamiento se centra en interrumpir la disfunción intracelular debida a los procesos de intoxicación que crea la presencia intra o extracelular de homotoxinas con un efecto desestabilizador dentro de la célula, por lo cual se debe integrar los tres fundamentos en la estrategia terapéutica (Broadfoot, 2006; IHA, 2007d).

Las fases de depósito e impregnación se caracterizan por períodos latentes de ausencia de signos clínicos, con el tiempo una enfermedad puede evolucionar desde la fase de excreción hasta la fase de desdiferenciación pasando desapercibida porque a veces no se presentan signos clínicos en el paso de una fase a la otra; la terapia antihomotóxica tiene como objetivo según sea el caso, combatir los síntomas, sustentar la función hepática, evitar daños por toxinas y actividad vírica, en cuyo caso aumenta la inmunidad celular (IHA, 2007a).

\section{MEDICAMENTOS ANTIHOMOTÓXICOS}

La homotoxicología contiene una gran variedad de fórmulas diseñadas por el Dr. Reckeweg y distribuido por diversos laboratorios, siendo el más representativo HEEL, que significa "herba est ex luce" (las hierbas son de la luz) (Broadfoot et al., 2009). Los medicamentos antihomotóxicos se utilizan basándose en el proceso fisiopatológico que padece el paciente, se componen normalmente de combinaciones de sustancias homeopáticas elaborados según las normas de la farmacopea homeopática alemana (HAB). La mayoría de los componentes que se utilizan en los medicamentos antihomotóxicos proceden de plantas y animales; 
además de eso se utilizan minerales e incluso catalizadores y cofactores. La terapia antihomotóxica es amplia y se divide en dos grandes grupos: Homeopáticos compuestos y unitarios (Cuadro 5), dentro de los primeros se encuentran: especialidades, composición, homaccord, nosodes organopreparados suis y en la segunda clasificación: catalizadores, injeels y medicamentos alopáticos homeopatizados (IHA, 2007c).

\section{DOSIFICACIÓN DE LOS MEDICAMENTOS HOMOTOXICOLÓGICOS EN MEDICINA VETERINARIA}

En principio se pueden utilizar los productos antihomotóxicos de dos formas: en función del tipo de enfermedad y su presentación aguda o crónica (García et al., 2003b), la dosificación de los medicamentos se hace tomado como referente, las fases subaguda o crónica de la enfermedad, se administran con menos frecuencia (una vez/día o a la semana), pero por un período más largo (4-6 semanas). Según García et al., (2003b) en estados agudos de la enfermedad se debe usar dosis frecuentes, en función de la gravedad e intensidad de las alteraciones, pudiéndose administrar medio a un comprimido, o 5 a 10 gotas cada 15 minutos durante un tiempo máximo de 2 horas, los medicamentos inyectables se pueden aplicar diariamente 1-2 veces, al observarse mejoría las frecuencias de administración se van ampliando progresivamente; en casos crónicos se puede emplear 1 comprimido o 10 gotas 10 veces al día, mientras que las dosis inyectables se administran $1-2$ veces por semana.

Autores como García et al., (2003b) hacen algunas recomendaciones para el uso de los medicamentos homotoxicológicos dependiendo la vía de administración:

- Vía intravenosa: en afecciones agudas aplicar con precaución.

- Vía intramuscular: actúa más lentamente, pero tiene efecto más duradero.

- Vía subcutánea: aplicada en zonas dolorosas o puntos de acupuntura tiene un efecto muy rápido.

- Vía oral: en los casos de gotas diluir en agua y beberlo en pequeñas cantidades durante el día. 
Cuadro 5. Medicamentos homotóxicos usados en medicina veterinaria y sus indicaciones

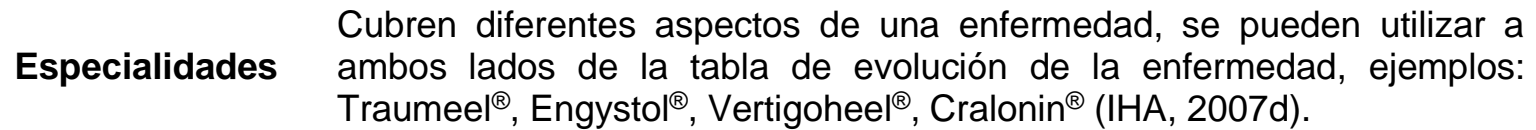

Tienen función protectora, detoxicadora, estimulante y aporte energético, aplicándose similitud y tropismo del órgano a tratar. En su fórmula se pueden encontrar nosodes, catalizadores, órgano-preparados, entre otros, se prescriben para tratar patologías crónicas y pacientes con evolución hacia la enfermedad (IHA, 2007g).

Son preparados de una o varias sustancias activas en acordes de potencia (diluciones altas, medias y bajas, de un mismo componente). Las diluciones bajas estimulan las funciones fisiológicas (hasta D8); las

Homaccord

Nosodes

Organopreparados suis

\section{Catalizadores}

Injeels

Medicamentos alopáticos homeopatizados medias regulan las funciones fisiológicas (D8-D30) y las altas (por encima de D30) tienen un efecto duradero e intenso; estos medicamentos vienen en forma de gotas orales o ampollas, ejemplo: Apis-Homaccord ${ }^{\circledR}$, Belladonna-HA ${ }^{\circledR}$, Colocynthis-HA®, Dulcamara-HA ${ }^{\circledR}$, Natrium-HA ${ }^{\circledR}$, Nux vomica-HA ${ }^{\circledR}(\mathrm{IHA}, 2007 \mathrm{f})$.

Son agentes patógenos inactivados por supreparación homeopática, sus mecanismos inmunológicos estimulan las reacciones terapéuticas (García et al., 2003b); se administran según principio de similitud, pero con $\mathrm{s}$ antihomotóxicos depurativos del mesénquima (Lymphomiosot ${ }^{\circledR} \mathrm{O}$ Galium-Heel ${ }^{\circledR}$ ) después de finalizar una enfermedad aguda previa, puesto que en fases agudas puede agravarse el paciente debido a que inducen más rápidamente la eliminación de las toxinas almacenadas en el mesénquima (IHA, 2007c).

Son para descubrir en un tejido u órgano las células defensivas de una inflamación, en enfermedades crónicas, ofrecen una posibilidad para la reactivación de funciones orgánicas; su mecanismo de acción se basa en el organotropismo de estimulantes. Su efecto no se observa en animales que presenten estados caquéxicos avanzados (IHA, 2007c).

Son elementos de la bioquímica celular que desbloquean un sistema enzimático (García et al., 2003b); intervienen en la respiración celular y obtención de energía. Su uso terapéutico es a nivel celular, por su alta estimulación, se recomienda que el animal tome un mínimo de 2-3 litros de agua durante los tres primeros días de tratamiento y suprimir la actividad física. Se usan en las fases celulares como paresia, neuralgias, úlcera gástrica y duodenal, pancreopatías, cirrosis hepática, durante y después de la irradiación con rayos X, entre otras (IHA, 2007c).

Son los medicamentos homeopáticos clásicos, y poseen un solo agente preparado homeopáticamente en una amplia variedad de diluciones (Broadfoot et al., 2009).

Se preparan a partir de antibióticos y analgésicos, entre otros; se utilizan para desintoxicar el organismo de dichos medicamentos y de los efectos que estos hayan podido o puedan causar (IHA, 2007c). 
Para la elección del tratamiento antihomotóxico en primer lugar se deben analizar las características (signos clínicos) y periodo de evolución de la enfermedad, puesto que todas las fases no se tratarán de la misma manera (García et al., 2003b); en forma general para las diferentes fórmulas homotoxicológicas, en presentación de gotas, se puede usar la siguiente dosificación:

- Perros grandes (mayor a $12.5 \mathrm{~kg}$ de peso) 10 gotas posología oral.

- Perros y gatos pequeños (menor a $12.5 \mathrm{~kg}$ de peso) 5 gotas posología oral.

En ambos casos la frecuencia de administración sigue los criterios dichos anteriormente, que depende de la fase de la enfermedad (aguda, crónica).

\section{CONCLUSIONES}

La medicina homotoxicológica tiene como objetivo terapéutico la estimulación y modulación de los mecanismos de defensa y reacciones orgánicas naturales, inducida por la utilización de sustancias homeopatizadas (minerales, vegetales y animales), teniendo en cuenta la función del órgano y tejido afectado, dando un enfoque diferente de los demás planes terapéuticos, por su mecanismo de acción basado en la reacción de asistencia inmunológica.

En el tratamiento homotoxicológico de cualquier patología hay que tener en cuenta que la misma es debida a desequilibrios predominantes en un nivel biológico, por lo tanto hay que observar los signos clínicos, realizar el examen clínico para llegar a un diagnóstico, apoyándose en pruebas paraclínicas (hemogramas, radiografías, ecografías y otros), como normalmente se procede en la medicina convencional, para luego determinar el uso de los medicamentos homotoxicológicos adecuados sin afectar negativamente los procesos fisiológicos normales del organismo.

En las patologías gastrointestinales en los animales de compañía tiene gran relevancia en la medicina homotoxicológica debido a que este sistema representa un medio de excreción constante de homotoxinas, siendo de gran importancia su adecuado tratamiento frente a la eliminación de toxinas. 


\section{REFERENCIAS BIBLIOGRÁFICAS}

1. Almanza J.E., Benavides O., Galán J. Análisis retrospectivo de las historias clínicas de una clínica veterinaria en Bogotá. NOVA Publicación en Ciencias Biomédicas. 5 (8): 168-176. 2007.

2. Angosto M.C. Bases celulares y moleculares de la regeneración hepática. Monografías del Instituto de España. 240 p. 2008.

3. Ares B.F., García A.D. Homotoxicología aplicada en el entorno quirúrgico; Homotoxicology Implemented in Surgical Setting. Revista Internacional de Ciencias Podológicas. 3 (2): 51-62. 2009.

4. Barros J. La inmuniestimulación en el paciente anciano según los métodos de la Medicina Biológica. 2011. Recuperado 17 Mayo 2016. Disponible En: http://medicinabiologica.eu/?p=327

5. Bastidas M.J.E. Efecto de árnica montana L. homeopatizada, en la regulación de citoquinas proinflamatorias y antiinflamatorias en cultivos celulares de linfocitos T humanos, MSc Medicina Alternativa - Homeopatía. Facultad de Medicina, Universidad Nacional de Colombia, Bogotá DC, Colombia. 55 p. 2012.

6. Brandan N.C., Llanos I., Ruiz D., Rodríguez A. Hormonas Catecolamínicas Adrenales. Cátedra de Bioquímica Facultad de Medicina. 2010. Recuperado $04 \quad$ Abril 2016.2 Disponible En: http://med.unne.edu.ar/sitio/multimedia/imagenes/ckfinder/files/files/CarreraMedicina/BIOQUIMICA/catecolaminas.pdf

7. Broadfoot P.J. Homotoxicology \& Chinese medicine - Rationales and rhythms: Scientific bases and support. Proceedings of the North American Veternary Conference. 20 (Junio 7-11): p 13-16. 2006.

8. Broadfoot P.J., Palmquist R.E., Johnston K., Wen J.J., Fougere B. Integrating complementary medicine into veterinary practice. John Wiley \& Sons, 2009.

9. Cian L.T., Homotoxicología: Una mirada hacia el futuro. Revista de la Facultad de Medicina, 10 (1). 2005. Recuperado 08 Septiembre 2016. Disponible En: http://terapianeuralveterinaria.blogspot.com.co/2008/06/homotoxicologiaunamirada-hacia-el.html

10. Condori C.A., Viscarra C., Zabaleta A.S. Homotoxicología. Revista Científica Ciencia Médica. 10 (1): 46-47. 2007.

11. Cooper D. Medicina biomoduladora u homotoxicología. Revista del Colegio de Nutricionistas. 6 (11): 39-40. 2010.

12. De Medio H. Veterinaria homeopática. Editorial Kier, Buenos Aires, Argentina. 416 p. 2004.

13. Fernández D.R., Brugieres L. Enfermedades del sistema mononuclear fagocítico. Histiocitosis. Medicine. 7: 2833-2837. 1997.

14. Fisher P., Dantas F. Homeopathic pathogenetic trials of acidum malicum and acidum ascorbicum. British Homoeopathic journal. 90 (3): 118-125. 2001.

15. García B.C., Benedito R.G., Calderón G.B., C. Principios fundamentales de la medicina homotoxicológica. canis et felis. (64): 57-71. 2003a. 
16. García B.C., Benedito R.G., Calderón G.B., C, Alarcón A.F. Enfoque terapéutico de la medicina homotoxicológica en veterinaria. canis et felis. (64): 73-85. 2003b.

17. Heine H., Schmolz M. Reacción de asistencia inmunológica mediante diluciones homeopáticas de extractos vegetales en los medicamentos antihomotóxicos. Biomedicina veterinaria. (2): 73-76. 1999.

18. Hernández U.M.A., Alvarado N.A. Interleucinas e inmunidad innata. Revista Biomédica. 12 (4): 272-280. 2001.

19. Herrera D.A.M., Villegas M.M.M. Influencia de la Coenzima ${ }^{\circledR}$ y el Ubichinon compositum $^{\circledR}$ sobre el ácido láctico, la frecuencia cardiaca y respiratoria en equinos pre post ejercicio en Bogotá, Médico Veterinario. Facultad de Medicina Veterinaria, Universidad de la Salle, Bogotá, Colombia. 133 p. 2005.

20. IHA, Academia Internacional de Homotoxicología, Drenaje y detoxificación. 2007a. Recuperado 17 Mayo 2016. Disponible En: http://www.iahonline.com/cms/docs/doc30774.pdf

21. IHA, Academia Internacional de Homotoxicología, Enfermedades digestivas. 2007b. Recuperado 15 Mayo 2016. Disponible En: http://www.iahonline.com/cms/docs/doc30778.pdf

22. IHA, Academia Internacional de Homotoxicología, Grupos de medicamentos en homotoxicología. 2007c. Recuperado 15 Mayo 2016. Disponible En: http://www.iah-online.com/cms/docs/doc30769.pdf

23. IHA, Academia Internacional de Homotoxicología, Introducción a la homotoxicología. 2007d. Recuperado 14 Mayo 2016. Disponible En: http://www.iah-online.com/cms/docs/doc30765.pdf

24. IHA, Academia Internacional de Homotoxicología, La matriz: histología y fisiología. 2007e. Recuperado 16 Mayo 2016. Disponible En: http://www.iahonline.com/cms/docs/doc30767.pdf

25. IHA, Academia Internacional de Homotoxicología, Los Homaccord. $2007 f$. Recuperado 17 Mayo 2016. Disponible En: http://www.iahonline.com/cms/docs/doc30772.pdf

26. IHA, Academia Internacional de Homotoxicología, Medicamentos "compositum". 2007g. Recuperado 17 Mayo 2017. Disponible En: http://www.iah-online.com/cms/docs/doc30771.pdf

27. IHA, Academia Internacional de Homotoxicología, Regulación orgánica. 2007h. Recuperado 15 Mayo 2016. Disponible En: http://iahonline.com/cms/docs/doc30776.pdf

28. IHA, Academia Internacional de Homotoxicología, Tipos de homotoxinas. 2007i. Recuperado 16 Mayo 2016. Disponible En: http://www.iahonline.com/cms/docs/doc30766.pdf

29. Murillo G.G. Consideraciones sobre algunos sistemas de medicina. Homeopatía-antipatía-isopatía-nihilismo terapéutico-alopatía. Medicina Interna de México. 26 (2): 155-166. 2010.

30. Smit A. Detoxificación y drenaje. Medicina biológica. 17 (1): 19-27. 2004.

31. Torres J. Que es la homotoxicología? 2009. Recuperado 16 Mayo 2016. Disponible En: http://cmbdrtorres.galeon.com/productos890358.html

32.Zepeda C.J. Resistencia de las bacterias a los antibióticos. Revista Médica Hondureña. 66 (2): 88-92. 1998. 